\title{
Intraoperative mapping and monitoring of brain functions for the resection of low-grade gliomas: technical considerations
}

\author{
Giulio Bertani, M.D., ${ }^{1}$ Enrica Fava, M.D., ${ }^{1}$ Giuseppe Casaceli, M.D., ${ }^{1}$ \\ Giorgio Carrabba, M.D., ${ }^{1}$ Alessandra Casarotti, Ph.D., ${ }^{1,2}$ Costanza Papagno, M.D., ${ }^{2}$ \\ Antonella Castellano, M.D., ${ }^{3}$ Andrea Falini, M.D., ${ }^{3}$ Sergio M. Gaini, M.D., ${ }^{1}$ \\ AND LoRenZo BELLO, M.D.'
}

${ }^{1}$ Division of Neurosurgery, Department of Neurological Sciences, Università degli Studi di Milano; ${ }^{2}$ Department of Psychology, University of Milano-Bicocca; and ${ }^{3}$ Department of Neuroradiology, University Vita e Salute, Istituto Scientifico, San Raffaele, Milan, Italy

\begin{abstract}
Low-grade gliomas ([LGGs] WHO Grade II) are slow-growing intrinsic cerebral lesions that diffusely infiltrate the brain parenchyma along white matter tracts and almost invariably show a progression toward malignancy. The treatment of these tumors forces the neurosurgeon to face uncommon difficulties and is still a subject of debate. At the authors' institution, resection is the first option in the treatment of LGGs. It requires the combined efforts of a multidisciplinary team of neurosurgeons, neuroradiologists, neuropsychologists, and neurophysiologists, who together contribute to the definition of the location, extension, and extent of functional involvement that a specific lesion has caused in a particular patient. In fact, each tumor induces specific modifications of the brain functional network, with high interindividual variability. This requires that each treatment plan is tailored to the characteristics of the tumor and of the patient. Consequently, surgery is performed according to functional and anatomical boundaries to achieve the maximal resection with maximal functional preservation. The identification of eloquent cerebral areas, which are involved in motor, language, memory, and visuospatial functions and have to be preserved during surgery, is performed through the intraoperative use of brain mapping techniques. The use of these techniques extends surgical indications and improves the extent of resection, while minimizing the postoperative morbidity and safeguarding the patient's quality of life.

In this paper the authors present their paradigm for the surgical treatment of LGGs, focusing on the intraoperative neurophysiological monitoring protocol as well as on the brain mapping technique. They briefly discuss the results that have been obtained at their institution since 2005 as well as the main critical points they have encountered when using this approach. (DOI: 10.3171/2009.8.FOCUS09137)
\end{abstract}

\section{KEY WoRdS - low-grade glioma • brain mapping • direct electrical stimulation $\quad$ intraoperative monitoring}

$\mathrm{R}$ ESECTION of LGGs, here understood as Grade II gliomas according to the WHO classification, ${ }^{67,68}$ forces the neurosurgeon to face uncommon difficulties and is still a subject of considerable debate. First, these tumors, which comprise Grade II astrocytoma (fibrillary and protoplasmic), Grade II oligoastrocytoma, and Grade II oligodendroglioma, are relatively rare; according to the Central Brain Tumor Registry of the Unit-

\footnotetext{
Abbreviations used in this paper: CST = corticospinal tract; CUSA = cavitron ultrasonic surgical aspirator; DES = direct electrical stimulation; DT $=$ diffusion tensor; $\mathrm{ECoG}=$ electrocorticography; $\mathrm{EEG}$ = electroencephalography; $\mathrm{EMG}$ = electromyography; $\mathrm{fMR}$ = functional MR; IFO = inferior frontooccipital fasciculus; ILF = inferior longitudinal fasciculus; LGG = low-grade glioma; $\mathrm{MEP}=$ motor evoked potential; SLF $=$ superior longitudinal fasciculus; $\mathrm{UNC}=$ uncinate fasciculus.
}

ed States, the various histological classes of LGGs have incidences varying between 0.10 and 0.46 per 100,000 population, and a cumulative incidence of $\sim 0.9$ per 100,000 people. By comparison, high-grade gliomas (WHO Grade III or IV) have a cumulative incidence of 3.7 per 100,000 population. ${ }^{22}$ Second, the tumors mainly affect young people (median age at diagnosis 35 years) and especially males (male/female ratio 1.5) with active professional and social lives. Third, LGGs present predominantly with seizures. ${ }^{82,105}$ Clinical symptoms are usually subtle, and the neurological examination findings are negative,${ }^{20}$ although these tumors tend to occur in eloquent areas. ${ }^{33}$ Lastly, some of these lesions may show an apparently slow pattern of growth. If the patients do not undergo appropriate serial MR imaging, it may be difficult to prove the tumor's volumetric increase. ${ }^{76}$ 


\section{G. Bertani et al.}

In the recent past, the aforementioned characteristics have caused a large part of the neurosurgical community to consider LGGs as indolent tumors, deserving a cautious and conservative diagnostic and therapeutic approach (wait and see, open/needle biopsy) to preserve the functional integrity of the patient. Currently, however, there is growing evidence that Grade II gliomas are aggressive neoplasms, with a constant growth rate of $\sim 4$ $\mathrm{mm} /$ year, ${ }^{76,86}$ which diffusely infiltrate the brain parenchyma along white matter tracts, ${ }^{73}$ and which almost invariably show a progression toward malignancy. ${ }^{33}$ They also frequently induce pharmacoresistent seizures, especially large and/or insular tumors. ${ }^{25,56,69}$ Furthermore, larger lesions are associated with a greater risk of malignant progression and with shorter patient survival. ${ }^{105}$ Finally, although LGGs have produced overt neurological deficits in only a few cases, they frequently present with more subtle symptoms affecting complex neurological functions (memory, language, character, visuospatial orientation, and so on), which require specific testing performed by a neuropsychologist to be detected. ${ }^{15,48-50,90}$

The rationale of the surgical treatment is based, first of all, on the ability to provide an adequate specimen for histological diagnosis and genetic and molecular analysis (1p19q loss of heterozygosity, MGMT methylation), ${ }^{24,57,60,106,112}$ whose results have fundamental therapeutic and prognostic implications. ${ }^{16,43,80,87,88,108,113}$ Moreover, surgery may contribute to control the frequency of seizures or to decrease the burden of drugs, ${ }^{14,25,39}$ as well as to improve the neurological symptoms that are directly related to the mass effect of the tumor on the cortex or on the functional fiber bundles. Finally, as shown by the most recent contributions in the literature, the extent of the resection is able to influence the overall survival and the time to malignant transformation. ${ }^{8,63,65,74,79,102,105}$ However, since the natural history of the tumor can be relatively long (with or without surgery), the conservation of simple and complex neurological functions of the patients is mandatory. ${ }^{10,27,32,94,105}$

For these reasons, at our institution resection is the first option in the treatment of LGGs, with the goal to maximally resect the tumor mass, while at the same time minimize the postoperative morbidity. This allows the patient's quality of life to be safeguarded and allows for prompt reintegration in professional and social lives, which is of primary importance considering the young age of the majority of these patients.

The identification of the eloquent cerebral areas, which are involved in motor, language, memory, and visuospatial functions and have to be preserved during surgery, is performed through the intraoperative use of brain mapping techniques. $5,7,10,12,32,35,40-42,53,105$

In this paper we present our paradigm for the surgical treatment of LGGs, focusing on the intraoperative monitoring protocol as well as on the brain mapping technique. In the end, we briefly discuss the results that have been obtained at our institution since 2005 as well as the main critical situations we have encountered using this approach.

\section{Methods}

\section{Patient Selection and Treatment Planning}

First of all we would like to stress the point that not all the patients who are affected by an LGG need brain mapping and awake craniotomy. A careful evaluation and selection of the patient is fundamental to customize the intraoperative mapping to the individual anatomofunctional characteristics of the patient and of the lesion. This goal is achieved throughout an extensive preoperative neurological, neuropsychological, and neuroradiological evaluation.

The presence of neurological deficits has to be carefully reported and quantified. Mild deficits are usually related to direct mass effect or infiltration of the tumor on the cortex or on the subcortical white matter tracts. In this case the surgeon may need to adapt the intraoperative stimulation parameters (for example, increasing the current intensity), or to perform an initial debulking of the tumor, to restore the normal excitability of the functional areas, as well as the response to direct stimulation. Complete deficits are extremely rare in this kind of lesion because the slow growth of these tumors allows a functional reorganization of the corticosubcortical network, known as brain plasticity, ${ }^{31,32}$ with transfer of the function to another area of the network. The presence of a complete deficit may suggest the interruption of an essential subcortical fiber bundle, as well as an aggressive behavior of the tumor.

As mentioned above, a neuropsychological evaluation is mandatory when considering surgery for this type of lesion because it allows detection of the subtle neurological alterations that are typical to each patient, to tailor the intraoperative testing to the patient and, postoperatively, to finely assess the impact of surgery on the patient's neurological functions. $1,2,55,70,71,111$ This examination should consist of as many tests as possible, to obtain an indepth analysis of the cognitive functions and of the emotional characteristics of the patient, and particularly of his language, memory and visuospatial abilities. This allows the individualization of the tasks to the intellectual and functional status of the patient. It is important to include in the battery both qualitative and quantitative tests, and normative data must be available for the quantitative procedure. It is also important that a speech therapist and/or a neuropsychologist be involved in managing the patient's assessments. Moreover, it is preferable that the same neuropsychologist performs the preoperative examination as well as the intraoperative testing. This enhances the coherence in the evaluation of the patient's answers and increases the reliability of the mapping. Also, the presence of a well-known person in the operating room reassures the patient and allows him to focus on the tests. ${ }^{115}$

The neuroradiological examination is composed of the usual MR imaging (T1-weighted, T2-weighted, FLAIR, and Gd-enhanced T1-weighted), and volumetric sequences, which are useful to measure the tumor volume and to determine its relationship with various structures,${ }^{77}$ such as major vessels. Further MR imaging studies include MR spectroscopy, which provides information on the metabolic characteristics of the tu- 


\section{Intraoperative mapping and monitoring of brain functions}

mor and allows for the design of a map of areas within the tumor in which the metabolism is more or less pronounced (multipixel MR spectroscopy map), ${ }^{45,52,72}$ and perfusion MR imaging studies, which may reveal areas of hyperperfusion, usually related to malignant transformation. ${ }^{23}$ At our institution, we also perform functional imaging, such as fMR imaging, and anatomical studies, such as DT imaging fiber tracking. We process fMR images with motor and language tasks, and use them mainly for the evaluation of the hemispheric dominance and for the perioperative surgical planning. ${ }^{13,58,114}$ The DT imaging fiber tracking maps give us a reconstruction of the subcortical fiber bundles involved in motor (CST), language (SLF, IFO, ILF, and UNC), and visuospatial functions, which run around or inside the tumor mass. ${ }^{3,6,11,19,26,51,59,84}$ These data are loaded into the neuronavigation system and help the surgeon in planning the resection, as well as in the performance of the cortical and subcortical mapping. ${ }^{61,100}$ It is worth mentioning that, although they actually reach a high level of accuracy in depicting the fiber bundles, tractography data are based on probabilistic measurements and, in the intraoperative setting, are affected by brain shift. ${ }^{62,64,83,89,91}$ For these reasons the information they provide is not sufficient to perform a safe and effective resection and must always be supported by the results of brain mapping. ${ }^{92}$ It is in fact the intraoperative mapping through DES which ultimately represents the gold standard for the identification and preservation of essential functional areas during surgery. These areas define the limit of resection, since their removal implies a high risk of permanent neurological deficits. 5,7,10,12,32,35,40,106

Once the preoperative workup is completed, which includes an anesthesiological examination, ${ }^{29,30}$ each patient is offered an individualized surgical and monitoring strategy based on the site and the characteristics of the tumor, the results of the neuropsychological evaluation and of the functional and anatomical imaging, ${ }^{110}$ which can be summarized as follows: 1) lesions in the nondominant hemisphere, away from eloquent areas and without relationship with areas of activation according to fMR imaging: motor monitoring (optional); 2) lesions in the nondominant hemisphere, in central or precentral area or in relationship with the CST (for example, insular and temporomesial tumors) and small central lesions in the dominant hemisphere: motor mapping and monitoring (awake anesthesia optional); 3) lesions in the nondominant hemisphere, in the postcentral region: motor mapping and monitoring, visuospatial mapping (awake anesthesia recommended); and 4) lesions in the dominant hemisphere: motor mapping and monitoring, language mapping and/ or visuospatial mapping for parietal lesions (awake anesthesia recommended).

From an anesthesiological point of view, besides the standard anesthesiological workup, the patient should be examined for his or her ability to be submitted to intraoperative awake monitoring when needed. It is recommended that preparation and selection of the patients be performed by anesthesiologists with expertise in awake surgery. ${ }^{29,30}$ At our institution, the only absolute contraindications to awake surgery, besides the lack of cooperation, are patient age $>65$ years, severe obesity, difficult airways, and severe cardiovascular or respiratory diseases.

When the patient requires only motor mapping, surgery is performed with the patient intubated through the nose, which allows the positioning of tongue and pharyngeal electrodes, and light surgical anesthesia is maintained throughout the procedure. No muscle relaxants are used during surgery to allow for neurophysiological assessment. When language or visuospatial functions have to be tested during surgery, the patient receives a laryngeal mask, which is maintained until after the craniotomy and opening of the dura. ${ }^{44}$ At this point, the patient is awakened, while adequate analgesia is maintained, for functional monitoring. Emesis is a rare complication and can be controlled by the administration of antiemetics at the beginning of this phase. ${ }^{78}$ The anesthesiologist should be able to keep the patient awake for the entire time of subcortical mapping, which may require, particularly during long-lasting operations, to alternate time for rest with periods during which the patient is fully awake and responsive. Total intravenous anesthesia with propofol and remifentanil is used at our institution for performing these procedures. ${ }^{28,103}$ However, newer drugs, such as dexmedetomidine, are emerging as effective and safe in producing sedation without inducing respiratory depression or affecting the electrophysiological monitoring. ${ }^{99,107}$ Unfortunately, dexmedetomidine currently still lacks approval for use in Europe.

\section{Intraoperative Protocol}

The intraoperative mapping and monitoring protocol, which is described in this work, has been used in at our institution during the last 4 years in $>400$ consecutive patients who underwent resection of gliomas located close or within motor, visuospatial, or language areas, or pathways. The majority of these cases were LGGs $(79.5 \%$, WHO Grade II), and the mean patient age was 36.4 years (range 16-68 years) (Table 1).

The major components of our neurophysiological protocol are EEG, ECoG, EMG, MEP, and DES techniques (Table 2). The protocol includes mapping and monitoring procedures. . $^{7,14,17,21,37,91,93,116}$

\section{Neurophysiological Techniques}

Electroencephalography/Electrocorticography. At our institution, EEG activity is recorded bilaterally by 4 subdermal needle electrodes, providing 4 bipolar leads. The EEG is registered to monitor brain activity when ECoG is not available, for example, at the beginning and end of surgery. Moreover, it allows assessment of brain activity away from the operating field, such as in the contralateral hemisphere.

The ECoG activity is recorded from a cortical region adjacent to the area being stimulated using subdural strip electrodes with 4-8 contacts, in a monopolar array, referred to a midfrontal electrode. Cerebral activity is recorded with a bandpass of $1.6-320 \mathrm{~Hz}$, and displayed with a sensitivity of $50-100 \mu \mathrm{m} / \mathrm{cm}$ for EEG and 200$400 \mu \mathrm{m} / \mathrm{cm}$ for ECoG. Continuous ECoG recordings (Comet, Grass) are used during the entire duration of the 


\section{G. Bertani et al.}

TABLE 1: Summary of clinical experience in 408 patients*

\begin{tabular}{|c|c|c|c|c|}
\hline \multirow[b]{2}{*}{ Location } & \multirow[b]{2}{*}{ No. of Patients } & \multirow[b]{2}{*}{ Type of Monitoring } & \multicolumn{2}{|c|}{ Deficits (no. of patients [\%]) } \\
\hline & & & Immediate & Permanent \\
\hline rolandic (nondominant) & 53 & $\begin{array}{l}\text { MEP, EEG, ECoG, DES (motor } \\
\text { mapping) }\end{array}$ & motor (32 [60.1]) & motor (1 [1.8]) \\
\hline rolandic (dominant) & 10 & & & \\
\hline small lesions $\dagger$ & 4 (all asleep) & $\begin{array}{l}\text { MEP, EEG, ECoG, DES (motor } \\
\text { mapping) }\end{array}$ & motor (4 [100]) & motor $(0[0])$ \\
\hline $\begin{array}{l}\text { medium/large } \\
\text { lesions }\end{array}$ & 6 (all awake) & $\begin{array}{l}\text { MEP, EEG, ECoG, DES (motor \& } \\
\text { language mapping) }\end{array}$ & motor (6 [100]); language (5 [83.3]) & motor (0 [0]); language (0 [0]) \\
\hline SMA (nondominant) & 23 & $\begin{array}{l}\text { MEP, EEG, ECoG, DES (motor } \\
\text { mapping) }\end{array}$ & motor (18 [78]) & motor $(0[0])$ \\
\hline SMA (dominant) & 31 (all awake) & $\begin{array}{l}\text { MEP, EEG, ECoG, DES (motor \& } \\
\text { language mapping) }\end{array}$ & $\begin{array}{l}\text { motor (26 [83.4]); language } \\
\quad(26 \text { [83.4]) }\end{array}$ & $\begin{array}{l}\text { motor (1 [3.2]]); language } \\
\quad(2[6.4])\end{array}$ \\
\hline frontal (nondominant) & 32 & MEP, EEG, EcoG (monitoring) & motor $(0[0])$ & motor $(0[0])$ \\
\hline frontal (dominant) & 92 (all awake) & $\begin{array}{l}\text { MEP, EEG, ECoG, DES (motor \& } \\
\text { language mapping) }\end{array}$ & $\begin{array}{l}\text { motor (30 [32.6]); language } \\
\quad(65[70.6])\end{array}$ & motor (0 [0]); language (2 [2.1]) \\
\hline temporal (dominant) & 101 (all awake) & $\begin{array}{l}\text { MEP, EEG, ECoG, DES (motor \& } \\
\text { language mapping) }\end{array}$ & motor (2 [1.9]); language (92 [91]) & motor (0 [0]); language (2 [1.9]) \\
\hline parietal (nondominant) & $\begin{array}{l}12 \text { (10 awake for } \\
\text { involvement of } \\
\text { 2nd branch of } \\
\text { SLF) }\end{array}$ & $\begin{array}{l}\text { MEP, EEG, ECoG, DES (motor } \\
\text { mapping; visuospatial mapping in } \\
\text { awakened) }\end{array}$ & $\begin{array}{l}\text { motor (3 [25]); visuospatial } \\
\quad(2[16.7])\end{array}$ & $\begin{array}{l}\text { motor (0 [0]); visuospatial } \\
\quad(0[0])\end{array}$ \\
\hline parietal (dominant) & 12 (all awake) & $\begin{array}{l}\text { MEP, EEG, ECoG, DES (motor, } \\
\text { language \& visuospatial mapping) }\end{array}$ & $\begin{array}{l}\text { motor (3 [25]); language (7 [58.3]); } \\
\text { visuospatial (2 [16.7]) }\end{array}$ & $\begin{array}{l}\text { motor (0 [0]); language } \\
\qquad(1[8.3]) ; \text { visuospatial (0 [0]) }\end{array}$ \\
\hline $\begin{array}{l}\text { paralimbic (non- } \\
\text { dominant) }\end{array}$ & 19 & $\begin{array}{l}\text { MEP, EEG, ECoG, DES (motor } \\
\text { mapping) }\end{array}$ & motor (4 [21.1]) & motor (1 [5.2]) \\
\hline paralimbic (dominant) & 23 (all awake) & $\begin{array}{l}\text { MEP, EEG, ECoG, DES (motor \& } \\
\text { language mapping) }\end{array}$ & $\begin{array}{l}\text { motor (2 [8.6]); language } \\
(17[73.9])\end{array}$ & motor (1 [4.3]); language (0 [0]) \\
\hline
\end{tabular}

procedure to monitor the brain basal electrical activity, to define the working current (detection of afterdischarges), and to monitor for the occurrence of afterdischarges, electrical seizures, or even clinical seizures during the resection (Fig. 1A). Because of this, EEG and ECoG recordings should be obtained during the entire duration of the operation. The occurrence of afterdischarges is quite common during these procedures, and the main objective of monitoring is to recognize those that occur in response to stimulation, to keep the reliability of the testing. Groups of ECoG spikes or electrical seizures occur in up to $30-40 \%$ of cases and can be related to the stimulation. In any case, when they appear, it is recommended that the cortex and the surgical cavity be irrigated with cold saline. In the majority of cases, this results in control and reversal of the situation. Clinical seizures occur in $8 \%$ of cases, and most of them are focal. The use of cold saline irrigation however, is able to control and totally revert most of them (Fig. 1B-D). In these cases, EEG is useful to detect diffusion of the seizure, either in the same or in the contralateral hemisphere. The few clinical seizures that we observed appeared most frequently at the end of the resection, when cortical stimulation was applied to assess the integrity of the motor pathway. The current was subsequently reduced, and no seizure was elicited anymore. This stresses the point that, at the end of the resection, it may be necessary to reduce the intensity of the current due to the reduction of the mass effect exerted by the tumor mass on the surrounding functional parenchyma. In selected cases, ECoG can be used to detect the generation of spikes in specific areas of the cortex, either close or far away from the tumor mass, which are responsible for sustained electrical activity.

Electromyography. Continuous multichannel EMG recording (Comet, Grass) is used throughout the duration of the procedure. Several separate muscles (agonist and antagonist muscles) can be monitored, either in the contralateral or ipsilateral body. Motor responses are collected by pairs of subdermal hooked needle electrodes inserted into the contralateral muscles from face to foot. Each pair of electrodes records 2 different muscles in the same body segment, to sample as many muscles as possible (that is, a flexor and an extensor muscle in the 


\section{Intraoperative mapping and monitoring of brain functions}

\section{TABLE 2: Neurophysiological protocol}

\begin{tabular}{|c|c|c|c|c|}
\hline Technique & Aim & Modality & Rationale & Indication \\
\hline EEG & $\begin{array}{l}\text { monitoring of basal } \\
\text { brain activity }\end{array}$ & $\begin{array}{l}\text { bilat recording, } 4 \text { bipolar } \\
\text { leads }\end{array}$ & $\begin{array}{l}\text { monitoring of brain areas not covered } \\
\text { by ECoG \& of the contralat hemi- } \\
\text { sphere, seizure surveillance at begin- } \\
\text { ning \& end of op; monitoring of level } \\
\text { of anesthesia }\end{array}$ & $\begin{array}{l}\text { each case undergoing cortical stimula- } \\
\text { tion (MEP, DES) }\end{array}$ \\
\hline ECoG & $\begin{array}{l}\text { direct monitoring of } \\
\text { cortical activity }\end{array}$ & $\begin{array}{l}\text { subdural strip/grid } \\
\text { electrodes, adjacent to } \\
\text { craniotomy site }\end{array}$ & $\begin{array}{l}\text { detection of afterdischarges, electrical/ } \\
\text { clinical seizures related to stimula- } \\
\text { tion, epileptogenic foci }\end{array}$ & $\begin{array}{l}\text { each case undergoing cortical stimula- } \\
\text { tion (MEP, DES), epilepsy op }\end{array}$ \\
\hline EMG & $\begin{array}{l}\text { monitoring/mapping of } \\
\text { motor activity }\end{array}$ & $\begin{array}{l}\text { subdermal hooked-needle } \\
\text { electrodes, extensive } \\
\text { insertion in several } \\
\text { contra- \& ipsilat muscles } \\
\text { from face to foot }\end{array}$ & $\begin{array}{l}\text { high sensitivity \& specificity in detecting } \\
\text { subclinical responses to MEP/DES, } \\
\text { allows the use of lower stimulation } \\
\text { intensity }\end{array}$ & $\begin{array}{l}\text { each case undergoing cortical \& sub- } \\
\text { cortical electrical stimulation, MEP }\end{array}$ \\
\hline MEP & $\begin{array}{l}\text { real-time, continuous } \\
\text { monitoring of motor } \\
\text { activity }\end{array}$ & $\begin{array}{l}\text { train of } 5 \text { technique, cortical } \\
\text { strip electrodes (never } \\
\text { transcranial) }\end{array}$ & $\begin{array}{l}\text { complementary to direct bipolar stimu- } \\
\text { lation, allows continuous monitoring } \\
\text { of motor pathways when DES is not } \\
\text { performed, can detect impending } \\
\text { brain ischemia }\end{array}$ & $\begin{array}{l}\text { identification of central sulcus \& moni- } \\
\text { toring of motor pathways }\end{array}$ \\
\hline DES & $\begin{array}{l}\text { mapping of cortical \& } \\
\text { subcortical functions }\end{array}$ & $\begin{array}{l}\text { direct electrical bipolar } \\
\text { stimulation }\end{array}$ & $\begin{array}{l}\text { allows direct identification of the cortical } \\
\qquad \& \text { subcortical functional areas }\end{array}$ & $\begin{array}{l}\text { accurate \& reliable testing of motor, } \\
\text { language, \& cognitive functions }\end{array}$ \\
\hline
\end{tabular}

forearm). On average, 16 channels are used for each procedure. The most used setting is that for the face (upper and lower face), neck, arm, forearm, hand, upper leg, and lower leg. A computerized video and image capturing system is continuously coupled with the EMG recordings, to further monitor and register the motor activity. In addition to EMG recordings, motor activity is also evaluated clinically.

Motor Evoked Potentials. Continuous monitoring of motor function is performed through MEP recording. The "train of five technique," which was introduced for surgery in anesthetized patients, has been described as sensitive in detecting imminent lesions of the motor cortex and the pyramidal pathways. ${ }^{109}$ For this purpose, a strip electrode containing 4-8 electrodes is placed over the precentral gyrus. In awake patients, a single stimulus or a double-pulse stimulus (individual pulse width $0.3-$ $0.5 \mathrm{msec}$, anodal constant current stimulation, interstimulus interval $4 \mathrm{msec}$, stimulation intensity close to motor threshold) is usually delivered. The muscle MEPs have to be recorded with either needle or, more conveniently in awake patients, with surface EMG electrodes. The MEP recording is usually alternated with direct cortical and subcortical motor mapping. Motor evoked potential monitoring is very useful because it provides real-time information on the integrity of the motor pathways during the resection of large parts of the tumor not closely related to the functional structures. In addition, MEPs provide warnings of impending brain ischemia due to critical vessel interruption, mostly in deep temporal or insular regions. ${ }^{81}$ Rarely, afterdischarges may occur also during
MEP monitoring (Fig. 1C), and occasionally partial seizures may occur (Fig. 1D).

Direct Electrical Stimulation. Direct electrical stimulation for cortical and subcortical mapping is performed by using a bipolar hand-held stimulator, ${ }^{9}$ with 1-mm electrode tips, $5 \mathrm{~mm}$ apart, connected to an Ojemann Cortical Stimulator (Integra Neuroscience) or an Osiris stimulator (Inomed). The stimulator delivers biphasic square wave pulses, each phase lasting $1 \mathrm{msec}$, at $60 \mathrm{~Hz}$ in trains lasting 1 second for cortical mapping and 1-2 seconds for subcortical mapping. Subcortical mapping is alternated with the resection in a back and forth manner. Subcortical mapping is performed using the same current threshold applied for cortical mapping.

When mapping is performed under general anesthesia, the current intensity ranges between 5 and $15 \mathrm{~mA}$, and the level of anesthesia, which strongly influences the excitability of the cortex, can be monitored using ECoG. In awake patients, a current intensity ranging between 2 and $8 \mathrm{~mA}$ is usually enough to evoke motor responses. In these patients, no electrodes are placed in the mouth, and the activity of the muscles in this region can be checked by monitoring the responses of the patients and by overt inspection. Awake patients are asked to relax before and during stimulation, and to assist in the description of induced movements or sensory changes.

The purpose of the mapping procedure is to reliably test motor, language, and cognitive function. At the beginning of the mapping procedure, the initial concern is to define the stimulation parameters. A low frequency of $60 \mathrm{~Hz}$ is used to establish the working current. It 


\section{G. Bertani et al.}

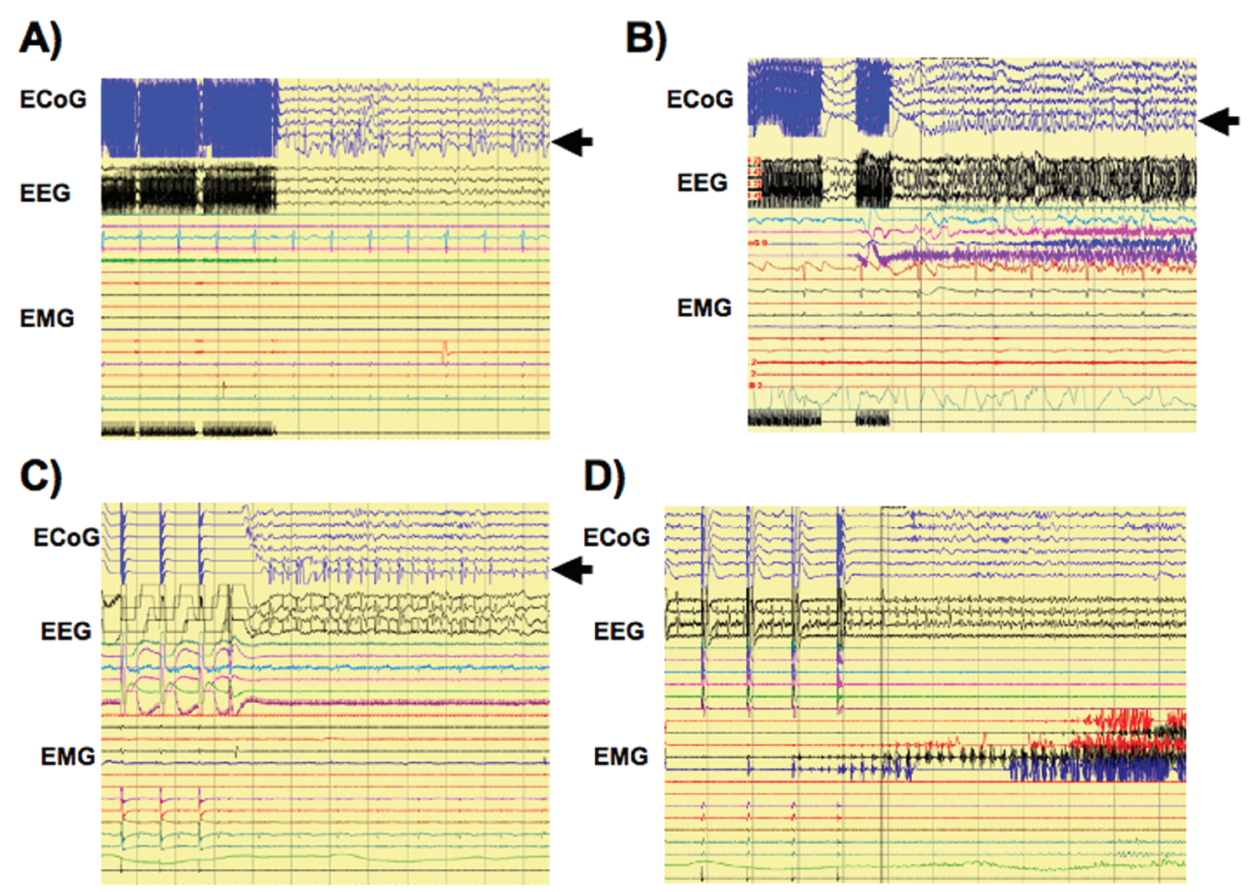

FIG. 1. Electrocorticography monitoring during cortical and subcortical mapping. A: Electrocorticography can be used to monitor the occurrence of afterdischarges. Afterdischarges (arrow) can appear as a single or a short train of spikes after the application of a stimulus. B: Occasionally, spikes can be organized (arrow), and a partial seizure may occur, as shown by EMG (upper and lower lips). The seizure was controlled by cold irrigation. $\quad \mathrm{C}$ and $\mathrm{D}$ : Rarely, single spikes may occur also during MEP monitoring (arrow in C) and occasionally also a partial seizure may occur (D), involving the hand and arm, as shown by EMG.

is advisable to start the procedure with the mapping of motor functions. Once determined, the same intensity of the stimulating current is used throughout the entire procedure in most cases, as well as for mapping cognitive and language functions. Initially, a low current intensity $(2 \mathrm{~mA})$ is used, and then progressively increased until a movement is induced. A stimulus duration of 1 or 2 seconds is usually enough to generate a motor response (Fig. 2A). At this point, it is good practice to stimulate the areas close to that in which the current induced the movement, to map them, and to check if the current is able to evoke motor responses also in these zones (Fig. 2B). If not, the current intensity may be increased and adjusted to evoke appreciable motor responses. It is also recommended to check the ECoG if the applied current induces afterdischarges in the nearby brain areas. In fact, only the current that is immediately below the one inducing the afterdischarges has to be used for mapping. If afterdischarges are seen, the current should be set up at least 0.5 $\mathrm{mA}$ lower than the previous one. Since only the responses evoked in absence of afterdischarges are considered to be trustworthy, continuous ECoG recording is used to check the appearance of afterdischarges during the mapping to keep the test reliable.

For language mapping, the initial test used is counting. The current is usually applied onto the premotor cortex related to the face, and the test is aimed to check if the current is able to stop the patient from counting. This has to be repeated several times, and the counting must be stopped at least 3 times to be reliable. ${ }^{85}$ If not, the current intensity is increased until this is produced. When the current is established, DES is applied to the entire exposed surface of the brain, and the occurrence of afterdischarges is checked using ECoG. The duration of the stimulus is between 3 and 4 seconds. Only the current that is not inducing afterdischarges in the entire stimulated cortex is used for mapping. In case of afterdischarges, the current intensity is decreased by at least $0.5 \mathrm{~mA}$.

It is important to keep the surface of the stimulated cortex moist and not to stop mapping after identifying only 1 eloquent site, but to search for possible redundancies; negative mapping does not protect but creates questionable stimulation reliability.

For subcortical mapping, either the same current used for cortical mapping or a current raised by $2 \mathrm{~mA}$ is applied, and the stimulus is continuously alternated with the resection (Fig. 3). Also during subcortical mapping, ECoG is continuously monitored to look for the occurrence of afterdischarges and seizures to verify the reliability of the responses. The resection margin is usually kept at least $5 \mathrm{~mm}$ away from functional areas and may come very close to subcortical pathways.

\section{Brain Mapping Techniques}

Motor Mapping. Electromyography recording provides an excellent view of the entire contralateral body, at the same time reducing the risk to miss responses in segments that are difficult to inspect due to the position of the patient on the operating table, or to detect areas such 


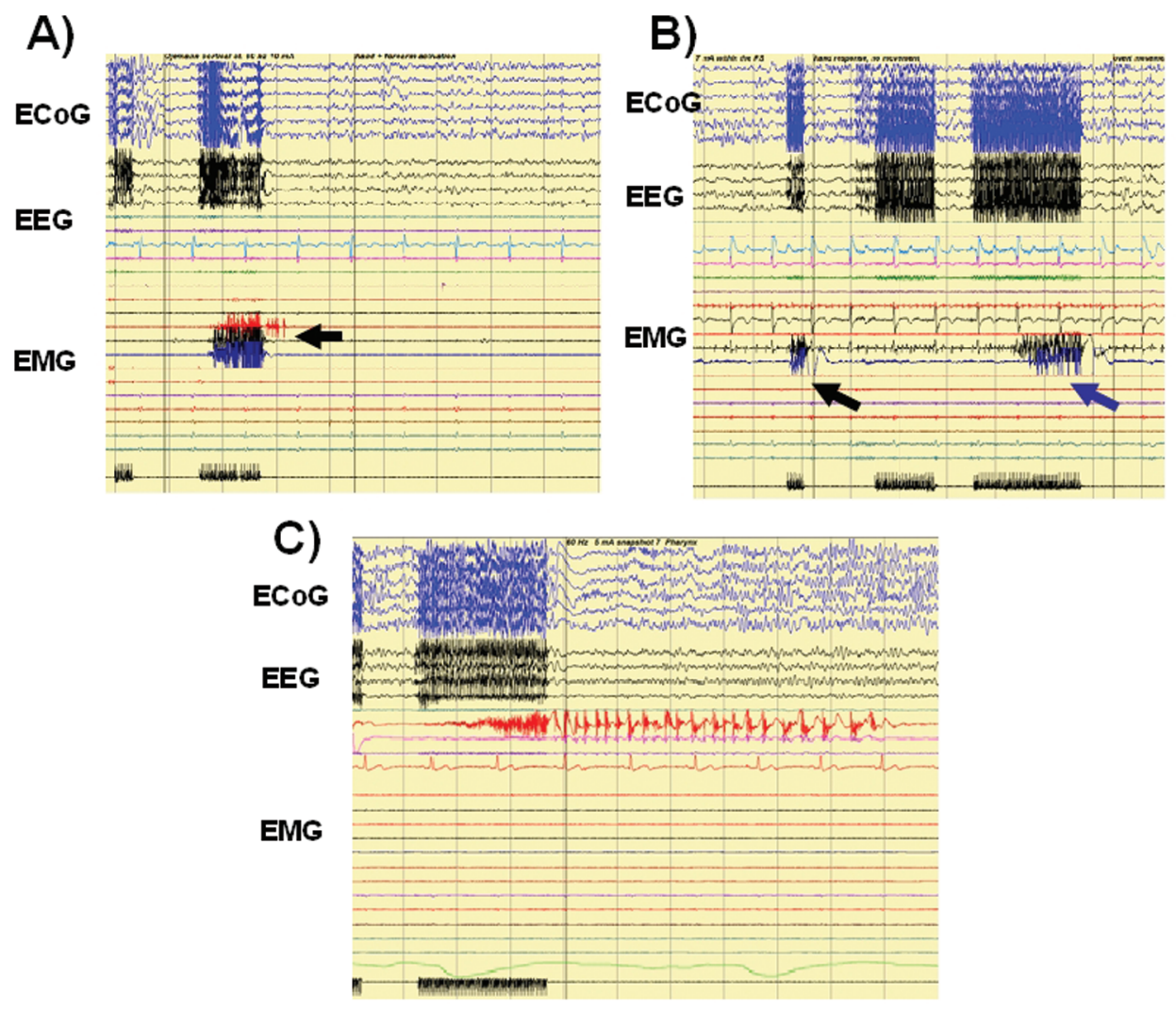

FIG. 2. Cortical motor mapping. The stimulus is applied over the area of the brain that is supposed to be the motor cortex. The stimulation of the motor cortex induces quite sharp tonic responses. Stimulation of this area allows for sequential identification of the areas of the brain whose stimulation evokes movements of the arm and hand (A), of the hand (black arrow), or of the hand and forearm (blue arrow) (B). Occasionally, a partial seizure may develop, which is controlled by cold saline irrigation (C).

as the mouth or the pharynx. In addition, even small amplitude responses, which do not evoke overt movements, can be detected (Fig. 3C). Occasionally, in patients under general anesthesia who are receiving a large number of antiepileptic medications, it might be difficult to evoke cortical motor responses. In these patients, MEP recording can be useful in identifying the location of the motor cortex and to plan the site of incision, allowing the resection to proceed. The same current intensity or one 2 $\mathrm{mA}$ higher is generally used for subcortical stimulation. Motor responses appear as focal (few muscles) when the tract is stimulated close to the surface, while they affect multiple muscle groups with deep stimulation. In addition, when the resection is approaching the deep portion of the tumor, subcortical stimulation allows small motor responses to be detected without overt muscle activity, which indicates that the resection is coming close to the motor pathway (Figs. 3 and 4). When these warning responses are identified, the resection should be performed particularly carefully, although it can proceed until more pronounced motor responses are identified, probably when the stimulator tip comes in direct contact with the CST. The identification of such warning signals is therefore particularly useful when performing a safe and effective resection.

The simultaneous use of CUSA and DES at the subcortical level near the pyramidal tract may lead to the abolition of previously evident motor responses. This abolition is generally reversible after turning the CUSA off. This interference with motor mapping may be interpreted as a transitory inhibition of axonal conduction. The clinical significance of this interference is relevant when CUSA and DES are used simultaneously, because it can decrease the sensitivity of the brain mapping technique, and it should be kept in mind by the surgeon when these tools are simultaneously used..$^{18}$

Language and Visuospatial Mapping. DES is delivered while the patient performs a series of language tests. The tests we most often administer are counting, object naming, famous face naming, and verb generation. To identify malcompliance or interference not related to the stimulation (for example, nonconvulsive seizures), each stimulation should be applied before the presentation of the object starts. Each stimulation should then be followed by at least a task without stimulation, and 2 tasks are the standard. Being the duration of the stimulation longer than that for motor mapping (4 seconds vs 1-2 seconds), repetitive stimulations carry a risk of triggering afterdischarges or seizures.

A dedicated neuropsychologist, who is present in the operating room and available for the entire mapping procedure, evaluates the patient performance during the various tests, both at the cortical and subcortical level. When 


\section{G. Bertani et al.}
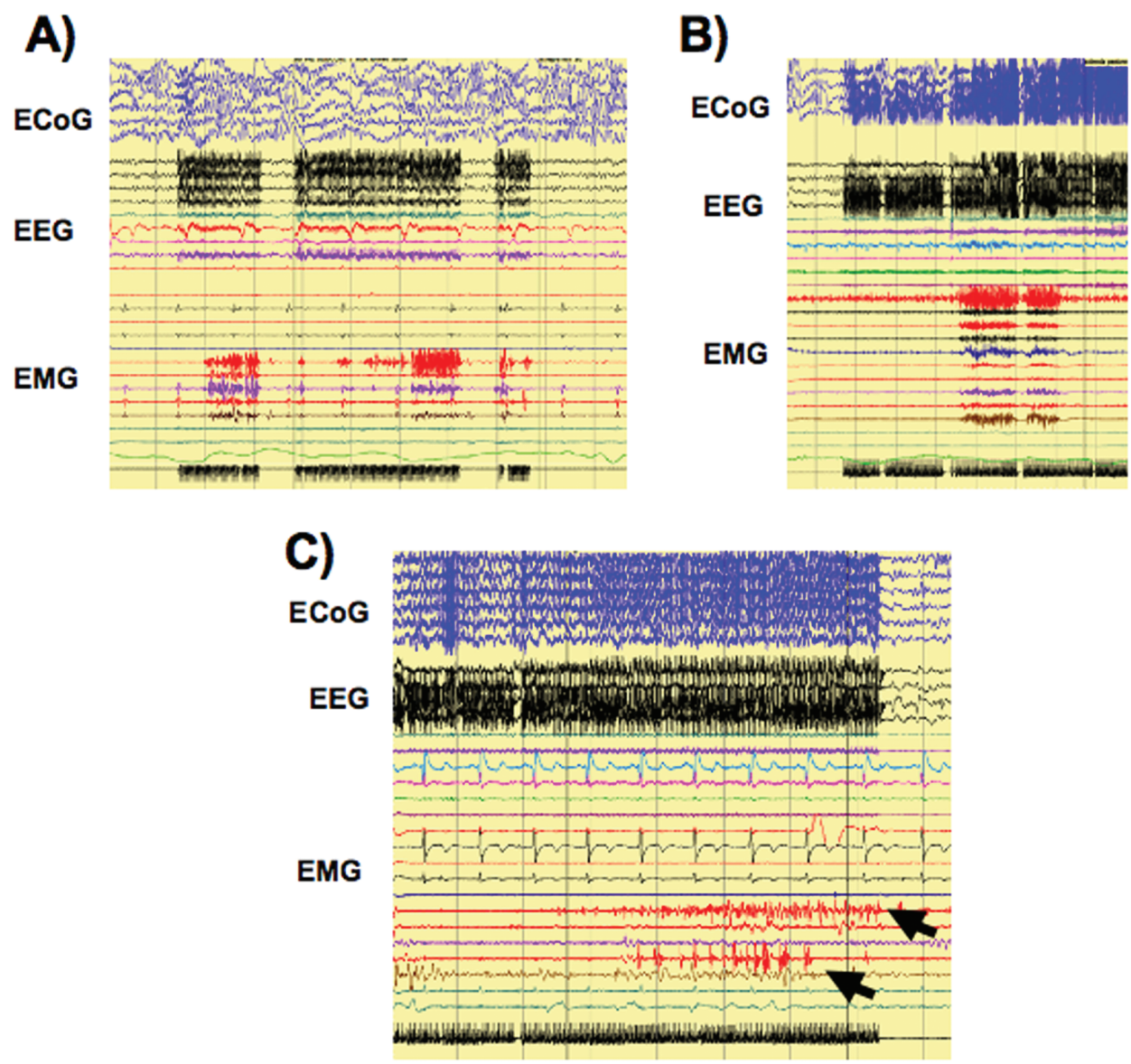

FIG. 3. Subcortical motor stimulation. Electromyography allows monitoring of the subcortical motor mapping responses. The stimulation of the peripheral portion of the CST induces evoked motor responses that involve various segments of the leg (A), and when the resection approaches the deepest part, the entire CST is stimulated (B). The use of EMG allows for identification of the motor tracts before overt motor responses are visible $(C)$. This is particularly useful in the deepest part of the resection cavity and reduces the risk of motor tract injury. The responses of the leg and foot were not clinically visible and were induced by the stimulation of the deepest portion of the resection cavity, close to the internal capsule.

a functional cortical or subcortical site related to the speech network is stimulated, the patient experiences a language disturbance, that is, he or she makes a mistake. Different kinds of mistakes may be encountered during the performance of the tests. ${ }^{38}$ These mistakes can occasionally occur without stimulation, or more frequently during the stimulation. It is important to check the ECoG and EEG during administration of each test for the occurrence of afterdischarges or electrical seizures. Only the mistakes made in absence of ECoG disturbances are reliable. In addition, a site can be defined as essential for language when it produces language disturbances at least 3 times in different nonconsecutive stimulations. ${ }^{85}$ Cortical language sites coding for object naming, verb generation, face naming, word or sentence comprehension, numbers, or colors can be identified in several regions in the frontal, temporal, or parietal lobe, with a distribution which differs according to the individual patient and the patient sex.

During subcortical language mapping, the patient is asked to perform object naming and verb generation tasks while the surgeon performs the resection, which is alternated with the stimulation. Each fiber tract is characterized by involvement in the semantic (IFO and UNC) or phonemic (SLF, ILF, and subcallosal fasciculus) loop and can be recognized at the subcortical level by the appearance of the language disturbances typical for each loop (for example, semantic paraphasias for IFO, phonemic paraphasias for SLF, and speech arrest for the subcallosal fasciculus). ${ }^{38}$ In the course of subcortical language mapping it is also possible to evoke motor responses, due to the stimulation of motor fibers belonging either to the premotor component of the face, which induces anarthria, or to the CST, which induces various type of muscle activation depending on the location and deepness of the stimulation (Fig. 5).

Visuospatial mapping is usually performed in patients with lesions located in the parietal lobe and, in case of dominant location, it is intermingled with language mapping (Fig. 6). The patients are usually asked to perform a line bisection task. Under DES, the patient is requested to look at the appearance of a line on a touch screen and to 

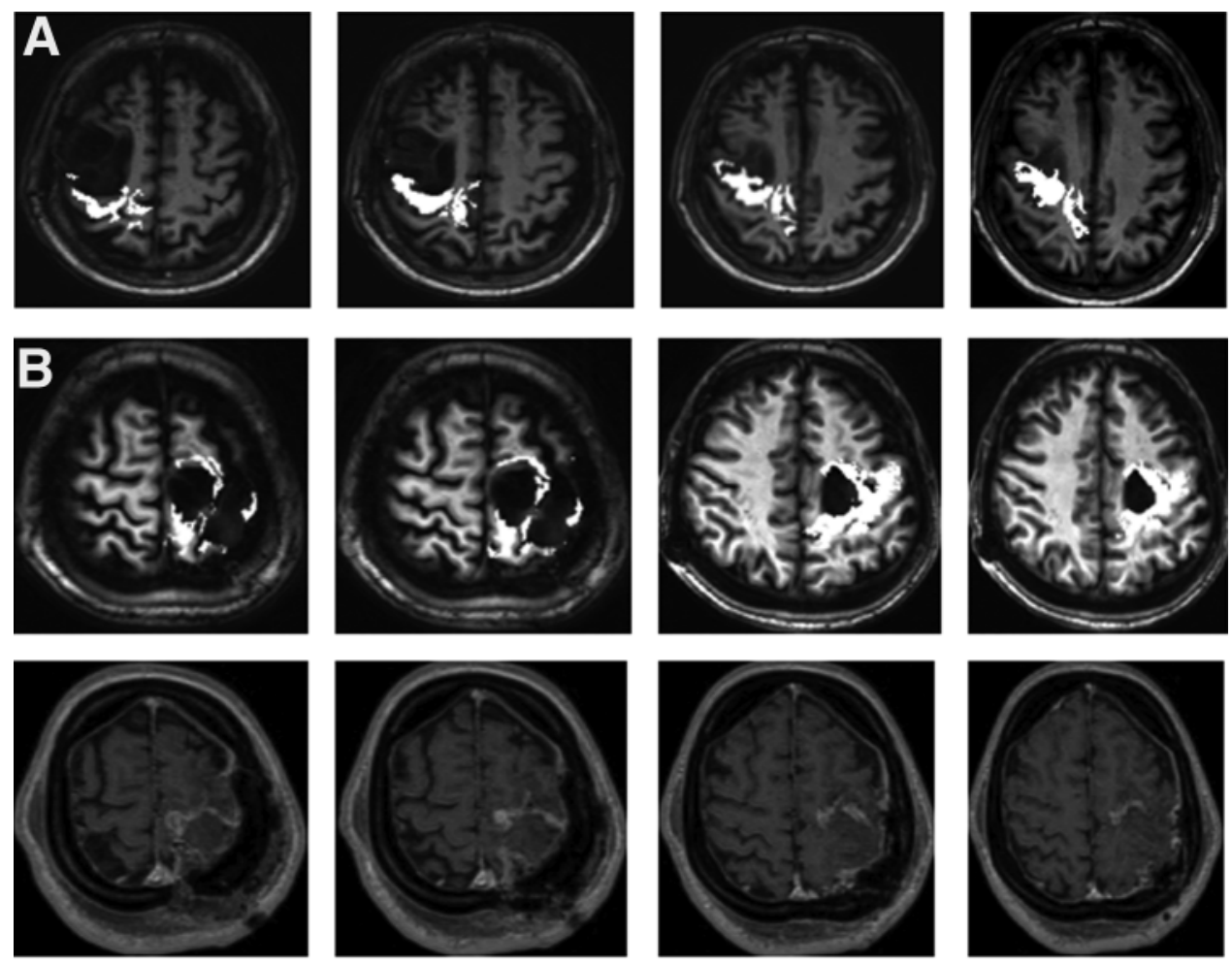

FIG. 4. Diffusion tensor imaging fiber tracking and subcortical motor mapping. When combined with subcortical mapping, DT imaging fiber tracking helps the surgeon in the safe identification of subcortical motor tracts. In this case of a central Grade II oligodendroglioma, the fiber bundles were located at the peripheral portion of the tumor (A and B upper, DT imaging fiber tracking reconstruction for CST overlaid on T1-weighted images). Removal was performed with the aid of subcortical motor mapping, during asleep anesthesia, because the tumor was confined within the CST. The resection was continued until functional margins were encountered. The resection margins are coincident with the functional motor tracts, as documented in the immediate postoperative postcontrast T1-weighted MR images (B lower).

bisect the line by touching its center with a pen. A deviation toward right or left of $>2 \mathrm{~cm}$ is usually considered pathological and is associated with an interference in the visuospatial function. The current intensity is the same as that for cortical motor mapping. One or two cortical sites are usually identified in all patients. The same procedure is also performed at the subcortical level by using the same current intensity or a current up to $2 \mathrm{~mA}$ higher the previous one. Subcortical visuospatial mapping can identify a small and discrete tract, usually running at the lateral mid border of the tumor, whose preservation, as well as that of the cortical sites, prevents the occurrence of neglect during the postoperative course.

\section{Intraoperative Imaging Techniques}

Intraoperative neuronavigation techniques, as mentioned above, are of great importance in our protocol. The neuronavigation system is loaded with morphological volumetric T1- and T2-weighted images, along with motor and language fMR and tractography images. Neuronavigation helps during surgery in localizing the tumor, and in defining the relationship between the tumor and the surrounding functional and anatomical structures, both at the cortical and the subcortical level. Functional and tractography data are also of valuable assistance as a guidance for the mapping procedure (Figs. 4-6). For an in-depth discussion of our intraoperative imaging protocol, please refer to a recent publication by our group. ${ }^{6}$

\section{Results}

\section{Functional Results}

Resection margins are usually kept $5 \mathrm{~mm}$ apart from essential cortical sites and are usually coincident with subcortical sites. When this is achieved, motor or language deficits develop in the immediate postoperative period in 72.8 and $65.4 \%$ of cases, respectively. When no subcortical sites are identified, this risk is very low (3$5 \%){ }^{5}$ In our experience, most of the deficits were transient and disappeared within 1 month after surgery. Overall, in the group of patients in which a subcortical functional site was identified during the resection, the likelihood of developing a permanent deficit was 3.8\%, independent of histology and location. This percentage reached $7 \%$ in patients with a preexisting motor or language deficit. In contrast, when no subcortical sites were found at the time of surgery, the chance to induce permanent deficits was even lower (2\%). These results further reinforce the concept that when a subcortical site is found, the surgeon is very close to the subcortical pathway. Therefore, when a subcortical response is reliably detected, the resection has to be stopped and should be continued in the adja- 


\section{G. Bertani et al.}
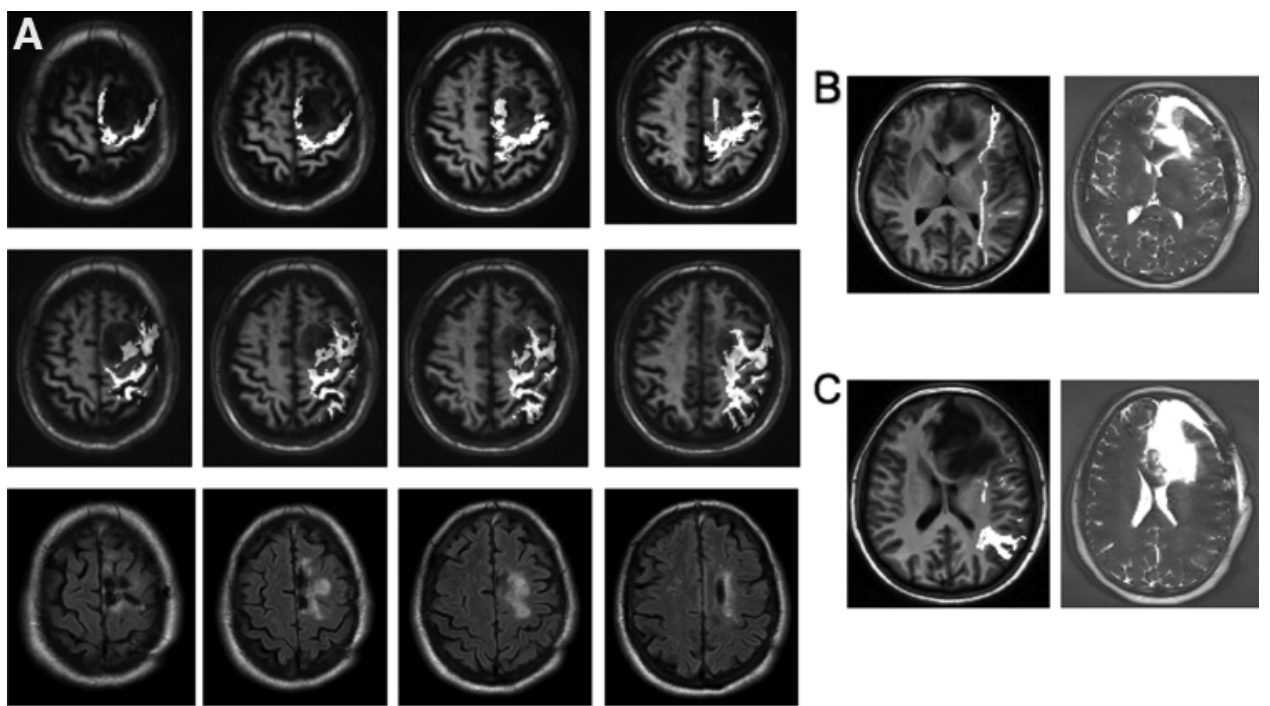

FIG. 5. Diffusion tensor imaging fiber tracking and subcortical language mapping. A: A low-grade left frontal oligodendroglioma. The DT fiber tracking images for CST (upper) and SLF (center) were merged with T1-weighted MR images and loaded into the neuronavigation system. The resection was performed using subcortical motor and language mapping and interrupted according to functional motor and language responses. The resection margins (lower), as shown on postoperative FLAIR images, are coincident with the functional language (SLF) and motor (CST) tracts. B and C: A left frontal F3 Grade II oligodendroglioma. The DT imaging fiber tracking reconstructions for IFO (B) and SLF (C) were fused with T1-weighted images (left panels). Both tracts constitute the lateral border of the tumor. Resection was performed using subcortical motor and language mapping, which were continuously alternated with the tumor resection. Resection was stopped when motor or language responses (phonemic paraphasias) were encountered. Postoperative T2-weighted images showing that the resection was performed according to the functional language tracts (IFO, SLF; right pane/s).

cent structures, because there is a high chance to damage functional structures. $5,39,66$ If no subcortical structures are found, the resection can be continued, because the chance to injure essential structures is low.

When we considered the results of the long-term postoperative neuropsychological evaluation, we found that $79.5 \%$ of the patients had a long-term postoperative normal language, $18.6 \%$ showed mild disturbances still compatible with normal daily life, and only $2.3 \%$ showed a long-term impairment. Similar figures were observed for the resection of gliomas close to motor areas or pathways.

As a final remark, at 3 months after surgery a high percentage of patients $(91.8 \%)$ returned to work.

\section{Oncological Results}

From an oncological point of view, firstly we managed to have a large amount of material for the histological and molecular diagnosis. In addition, the first documented oncological result of a surgical approach performed with the aid of brain mapping techniques was the increase in the number of cases which were submitted to surgical treatment: in accordance with what has been previously reported in the literature, this percentage in our series increased from $11 \%$ of cases, when mapping was not available, to $81 \%$ when mapping was applied, with a significant decrease in the number of cases in which biopsy alone was performed. $5,31,34,36,40$ The second oncological result, already mentioned in the previous paragraph, is the decrease in the percentage of postoperative permanent deficits, which decreased from 33 to
$2.3 \%$, either for language or motor function. A third important effect is the decrease in the incidence of seizures, particularly in patients with a long epileptic history and affected by insular tumors. Lastly, and most important, is the impact that these techniques have on the extent of resection. The use of brain mapping techniques increased the percentage of patients in whom a total and subtotal resection was achieved. In our series, the percentage of total and subtotal resections increased from $11 \%$, in the period in which no mapping was available, ${ }^{5}$ to $69.8 \%$ in the era in which brain mapping techniques were applied. These figures are in accordance with the results of other groups. ${ }^{65,92,102,105}$

\section{Discussion}

The purpose of brain mapping techniques is to identify and preserve the cortical and subcortical sites at the time of surgery that are essential in maintaining function. In our experience, motor or language disturbances associated with most LGGs can be induced either inside the tumor mass or at the tumor margins, because most of the essential sites, particularly at the subcortical level, are located within the tumor or adjacent to it. The resection was stopped when language, motor, visuospatial, cortical, or subcortical areas were encountered. This allowed us to have an extremely low percentage of postoperative permanent neurological deficits. In particular, the preservation of subcortical tracts seemed to be critical for the long-term patient integrity. 5,6,10,35,39,40 The fact that most of the deficits were transient suggests that in these cases the resection was conducted close to the tracts, and that 

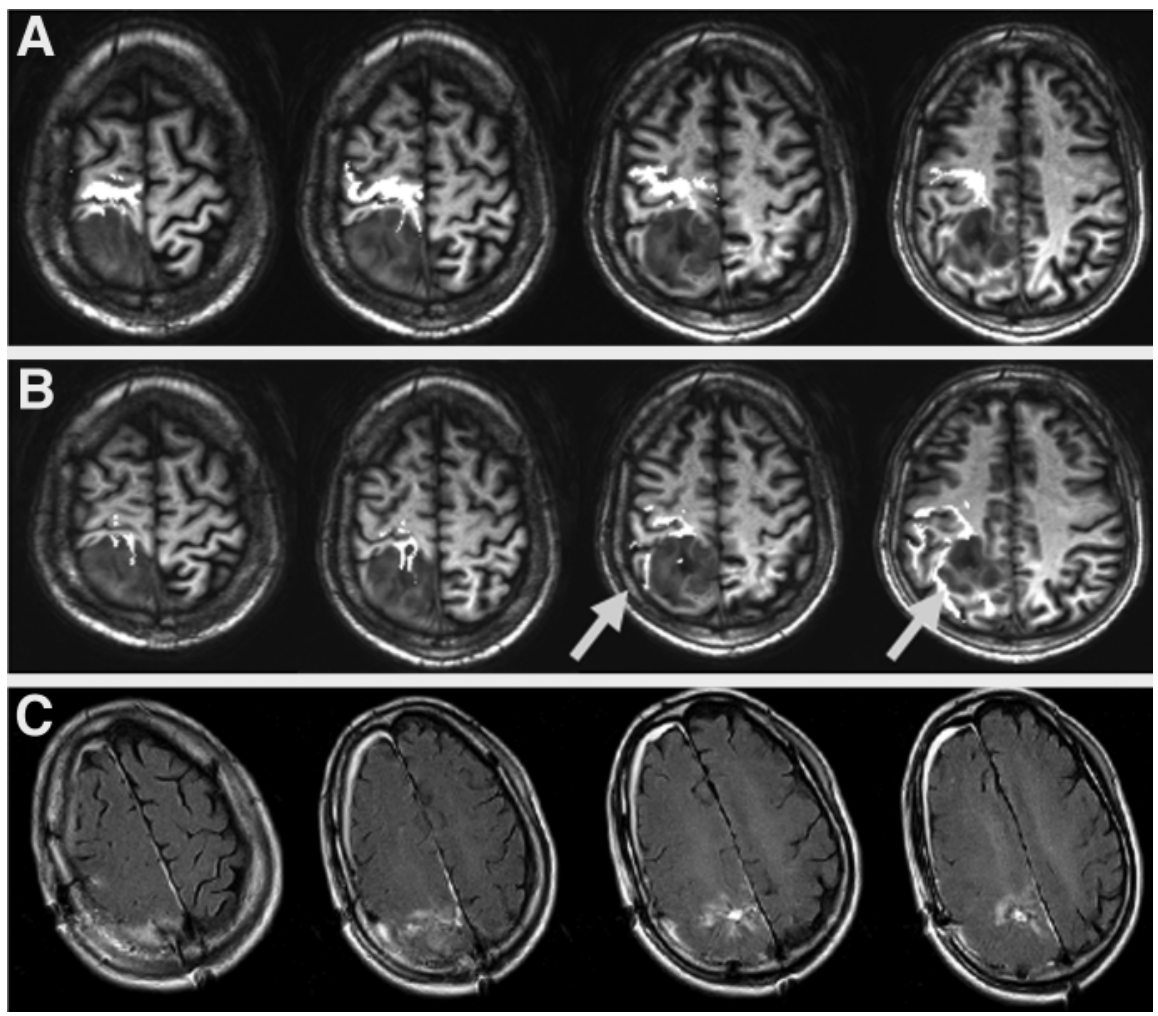

FIG. 6. Diffusion tensor imaging fiber tracking motor and visuospatial mapping. A right parietal low-grade oligodendroglioma in which motor and visuospatial mapping were performed to aid resection. The DT fiber tracking images for CST (A) and SLF (B) were merged with T1-weighted MR images and loaded into the neuronavigation system. Both series show that CST and SLF (the second branch is indicated with arrows) are at the tumor border. Resection was performed with the aid of subcortical motor mapping to define the anterior margin of resection, which was stopped when motor responses were encountered. Resection was also performed with the aid of visuospatial mapping to define the lateral and posterior margin of the tumor, defined by the second branch of the SLF. In this case, the patient was awakened and asked to touch the center of a line on a touch screen, during subcortical stimulation. A deviation toward right of $>2 \mathrm{~cm}$ is usually considered as pathological, and associated with an interference in the visuospatial function. Postoperative FLAIR images (C) indicate that resection margins were coincident with functional tracts.

the development of postoperative disturbances may have been due to edema or transient retraction injury, without permanent damage. The low incidence of postoperative deficits in patients in whom no subcortical tracts were identified was usually due to vascular damage and development of ischemic areas. The MEP monitoring can help in preventing the appearance of motor deficits due to vascular injury. ${ }^{81}$ In addition, our functional results were totally different from those obtained when DES was not applied. The analysis of patients with high-grade gliomas or LGGs who underwent surgery at our institution before the use of direct electrical stimulation, showed $23 \%$ of permanent language or motor deficits, in accordance with what has been previously reported in other series. ${ }^{5,31,40}$ In addition, brain mapping did not negatively affect our ability to perform an extensive resection in a large percentage of cases. On the contrary, the percentage of total and subtotal resections significantly improved in comparison with the time in which DES was not applied. Recent evidence from other groups has shown that a more extensive resection at the time of the initial diagnosis may be a favorable prognostic factor for this type of tumor, and has an influence on time to recurrence and malignant transformation.
Accordingly, an influence on patient survival has been documented as well. ${ }^{65,92,102,105}$ This stresses the point that the smaller the tumor, the better the patient outcome. As a consequence, delaying surgical intervention may increase the risk of malignant transformation. Altogether, these data support resection as the first choice of treatment for LGGs and show the relevance of cortical and subcortical stimulation as an extremely useful surgical adjunct during the removal of lesions involving eloquent areas.

Nevertheless, brain cortical and subcortical mapping is a demanding technique. Especially during awake surgery, it requires a close collaboration among the neurosurgeon, neuropsychologist, and neurophysiologist. The neuropsychologist and neurophysiologist should both be present in the operating room and work as a team to assist the surgeon in combining the neurophysiological information with the interpretation of the language disturbances, and to compare these data with the surgical anatomy. In addition, a well-trained anesthesiologist is essential, because he or she must titrate the sedation and the analgesics to keep the patient not only calm and pain free but also fully awake and able to reliably perform the tasks. Either excessive sedation or anxiety and pain, in fact, may 


\section{G. Bertani et al.}

reduce the compliance of the patient and compromise the results of the tests. It is also worth mentioning that, in case of awake anesthesia, the patient needs to be prepared in advance to the awakening phase and to the performance of the tasks in the operating room. In fact, these events can be particularly stressful, and only patients who are properly instructed and motivated are able to tolerate the operative environment and to focus on the tests. ${ }^{115}$ During mapping, a high level of interaction among the operating team is key to obtaining an accurate evaluation of each task and a precise definition of the functional sites. Extensive training of each team member and a habit of working together are important to spare time and assist the surgeon with clear indications. Brain mapping can be a significantly time-consuming procedure, in particular when the results of mapping are unsatisfactory and additional or repeated testing is required. This may obviously result in supplementary burden for the patient and need of rest, with further prolongation of surgical times. Moreover, a tired patient is prone to performing poorly on the tests. If the patient's compliance is compromised because of an especially lengthy procedure, it is recommended to stop surgery and to plan a second intervention in 2-3 months. This can also be scheduled in advance, based on the preoperative tumor size and characteristics. ${ }^{104}$

Along with the aforementioned issues, brain mapping is intrinsically limited by the fact that only the functions that are specifically tested are preserved. If this is of relative importance for simple functions, such as motor function, it is particularly relevant for complex cognitive functions. As already mentioned, time strongly affects the quality of mapping and that means that only a limited number of well-selected tests can be administered to the patient. This should be kept in mind when dealing with large tumors located in the dominant hemisphere in areas densely filled with functional sites, such as the temporoparietal junction or the precentral area. ${ }^{46,101}$ In such regions, careful selection of the tasks and a systematic execution of the mapping are crucial to save the basic cognitive functions, but may not be able to investigate other superior functions, such as calculation, writing, reading, and second languages. ${ }^{4,32,47,75,95-98}$ Therefore, the surgeon has to plan preoperatively, according to tumor size and location, which information should be obtained through the mapping procedure, and to inform the patient about the possible limitations of each approach.

Another technical critical issue is the relationship between the intensity of the stimulating current and the distance from the functional site, in particular when subcortical mapping is performed. In the literature there are no available works studying the penetration distance of subcortical bipolar stimulation in the white matter, while the range of bipolar stimulation on the cortex has been observed to be $\sim 2-10 \mathrm{~mm} .{ }^{11,54}$ In our experience, when a response was induced at a subcortical level, we always performed an intensity-response curve to assess the maintenance of the response either at very low current intensity levels. This can help in estimating the distance between the point of stimulation and the functional tract. When we reached the functional fibers, we always stopped the resection, managing to preserve the function- al pathways in the large majority of cases. In addition, we commonly observed that, as we approximated to the end of resection, a lower current intensity was needed to induce a response. On the other hand, as already mentioned, applying the same current intensity used at the beginning of resection induces more marked responses and carries a higher risk of inducing seizures. Probably, functional structures regain their normal excitability threshold once the mass effect exerted by the tumor is relieved. Anesthesiological factors may also play a role (for example, progressive clearance of anesthetic drugs). To keep the reliability of the mapping and to avoid false-positive findings, which could lead to premature interruption of the resection, it is recommended that the working current be verified and decreased once a large part of the tumor has been removed. Nevertheless, further studies are needed to clarify this point.

\section{Conclusions}

Low-grade gliomas are slow-growing intrinsic lesions that diffusely infiltrate the brain parenchyma along white matter tracts, and that almost invariably show a progression toward malignancy. At our institution, resection represents the first option in the treatment of LGGs. Surgical removal of these lesions requires the combined efforts of a multidisclipinary team of neurosurgeons, neuroradiologists, neuropsychologists, and neurophysiologists, who together contribute to the definition of the location, extension, and extent of functional involvement that a specific lesion has induced in a particular patient. It is important to keep in mind that each tumor has caused specific changes to the brain functional network, with high interindividual variability. This requires that each treatment plan is tailored to the tumor and to the patient. When this is accomplished, surgery should be performed according to functional and anatomical boundaries, aiming to maximally resect the mass and to maximally preserve the functional integrity of the patient. This can be reached at the time of the initial surgery, depending on the functional organization of the brain, or may require additional surgeries. The use of so-called brain mapping techniques extends surgical indications and improves the extent of resection, with minimization of morbidity and an increase in quality of life. However, because of the complexity of this approach, extensive training and the formation of a dedicated team are essential.

\section{Disclosure}

This work was supported by grants from AIRC (Associazione Italiana Ricerca sul Cancro) and Fondazione Italo Monzino to Dr. Bello.

\section{References}

1. Andrewes DG, Kaye A, Aitken S, Parr C, Bates L, Murphy M: The ESDQ: a new method of assessing emotional and social dysfunction in patients following brain surgery. J Clin Exp Neuropsychol 25:173-189, 2003

2. Andrewes DG, Kaye A, Murphy M, Harris B, Aitken S, Parr $\mathrm{C}$, et al: Emotional and social dysfunction in patients following surgical treatment for brain tumor. J Clin Neurosci 10:428-433, 2003 
3. Basser PJ, Pajevic S, Pierpaoli C, Duda J, Aldroubi A: In vivo fiber tractography using DT-MRI data. Magn Reson Med 44:625-632, 2000

4. Bello L, Acerbi F, Giussani C, Baratta P, Taccone P, Songa $\mathrm{V}$, et al: Intraoperative language localization in multilingual patients with gliomas. Neurosurgery 59:115-125, 2006

5. Bello L, Gallucci M, Fava M, Carrabba G, Giussani C, Acerbi $\mathrm{F}$, et al: Intraoperative subcortical language tract mapping guides surgical removal of gliomas involving speech areas. Neurosurgery 60:67-82, 2007

6. Bello L, Gambini A, Castellano A, Carrabba G, Acerbi F, Fava E, et al: Motor and language DTI Fiber Tracking combined with intraoperative subcortical mapping for surgical removal of gliomas. Neuroimage 39:369-382, 2008

7. Berger MS: Functional mapping-guided resection of lowgrade gliomas. Clin Neurosurg 42:437-452, 1995

8. Berger MS, Deliganis AV, Dobbins J, Keles GE: The effect of extent of resection on recurrence in patients with low grade cerebral hemisphere gliomas. Cancer 74:1784-1791, 1994

9. Berger MS, Ojemann GA, Lettich E: Neurophysiological monitoring during astrocytoma surgery. Neurosurg Clin $\mathbf{N}$ Am 1:65-70, 1990

10. Berger MS, Rostomily RC: Low grade gliomas. Functional mapping resection strategies, extent of resection, and outcome. J Neurooncol 34:85-101, 1997

11. Berman JI, Berger MS, Mukherjee P, Henry RG: Diffusiontensor imaging-guided tracking of fibers of the pyramidal tract combined with intraoperative cortical stimulation mapping in patients with gliomas. J Neurosurg 101:66-72, 2004

12. Black PM, Ronner SF: Cortical mapping for defining the limits of tumor resection. Neurosurgery 20:914-919, 1987

13. Bogomolny DL, Petrovich NM, Hou BL, Peck KK, Kim MJ, Holodny AI: Functional MRI in the brain tumor patient. Top Magn Reson Imaging 15:325-335, 2004

14. Branco DM, Coelho TM, Branco BM, Schmidt L, Calcagnotto ME, Portuguez M, et al: Functional variability of the human cortical motor map: electrical stimulation findings in perirolandic epilepsy surgery. J Clin Neurophysiol 20:17-25, 2003

15. Brown PD, Buckner JC, O'Fallon JR, Iturria NL, O'Neill BP, Brown CA, et al: Importance of baseline mini-mental state examination as a prognostic factor for patients with low-grade glioma. Int J Radiat Oncol Biol Phys 59:117-125, 2004

16. Capelle L, Duffau H, Lopes M, Sichez JP, Bitar A, Faillot T, et al: Who grade 2 gliomas in adults: a study of prognostic factors with special emphasis on the role of surgery. J Neurooncol 4:S17-S69, 2002

17. Carrabba G, Fava E, Giussani C, Acerbi F, Portaluri F, Songa $\mathrm{V}$, et al: Cortical and subcortical motor mapping in rolandic and perirolandic glioma surgery: impact on postoperative morbidity and extent of resection. J Neurosurg Sci 51:45-51, 2007

18. Carrabba G, Fava E, Mandonnet E, Capelle L, Duffau H, Bello L: Transient axonal inhibition induced by CUSA during brain mapping: a case report with motor EMG evidence. Neurosurgery 63:E178-E179, 2008

19. Catani M, Howard RJ, Pajevic S, Jones DK: Virtual in vivo interactive dissection of white matter fasciculi in the human brain. Neuroimage 17:77-94, 2002

20. Cavaliere R, Lopes MB, Schiff D: Low-grade gliomas: an update on pathology and therapy. Lancet Neurol 4:760-770, 2005

21. Cedzich C, Taniguchi M, Schaffer S, Schramm J: Somatosensory evoked potential phase reversal and direct motor cortex stimulation during surgery in and around the central region. Technical application. Neurosurgery 38:962-971, 1996

22. Central Brain Tumor Registry of the United States: Statistical report: Primary Brain Tumors in the United States, 2000-
2004. (http://www.cbtrus.org/reports//2007-2008/2007report. pdf) [Accessed September 2, 2009]

23. Cha S, Tihan T, Crawford F, Fischbein NJ, Chang S, Bollen A, et al: Differentiation of low-grade oligodendrogliomas from low-grade astrocytomas by using quantitative blood-volume measurements derived from dynamic susceptibility contrastenhanced MR imaging. AJNR Am J Neuroradiol 26:266273, 2005

24. Chahlavi A, Kanner A, Peereboom D, Staugaitis SM, Elson P, Barnett G: Impact of chromosome 1p status in response of oligodendroglioma to temozolomide: preliminary results. J Neurooncol 61:267-273, 2003

25. Chang EF, Potts MB, Keles GE, Lamborn KR, Chang SM, Barbaro NM, et al: Seizure characteristics and control following resection in 332 patients with low-grade gliomas. J Neurosurg 108:227-235, 2008

26. Clark CA, Barrick TR, Murphy MM, Bell BA: White matter fiber tracking in patients with space-occupying lesions of the brain: A new technique for neurosurgical planning? Neuroimage 20:1601-1608, 2003

27. Claus EB, Horlacher A, Hsu L, Schwartz RB, Dello-Iacono D, Talos F, et al: Survival rates in patients with low-grade glioma after intraoperative magnetic resonance image guidance. Cancer 103:1227-1233, 2005

28. Conte V, Baratta P, Tomaselli P, Songa V, Magni L, Stocchetti N: Awake neurosurgery: an update. Minerva Anestesiol 74:289-292, 2008

29. Danks RA, Aglio LS, Gugino LD, Black PM: Craniotomy under local anesthesia and monitored conscious sedation for the resection of tumors involving eloquent cortex. J Neurooncol 49:131-139, 2000

30. Danks RA, Rogers M, Aglio LS, Gugino LD, Black PM: Patient tolerance of craniotomy performed with the patient under local anesthesia and monitored conscious sedation. Neurosurgery 42:28-36, 1998

31. Duffau H: Lessons from brain mapping in surgery for lowgrade glioma: insights into associations between tumour and brain plasticity. Lancet Neurol 4:476-486, 2005

32. Duffau H: New concepts in surgery of WHO grade II gliomas: functional brain mapping, connectionism and plasticity-a review. J Neurooncol 79:77-115, 2006

33. Duffau H, Capelle L: Preferential brain locations of low-grade gliomas. Cancer 100:2622-2626, 2004

34. Duffau H, Capelle L, Sichez J, Faillot T, Abdennour L, Law Koune JD, et al: Intraoperative direct electrical stimulations of the central nervous system: the Salpetriere experience with 60 patients. Acta Neurochir (Wien) 141:1157-1167, 1999

35. Duffau H, Capelle L, Sichez N, Denvil D, Lopes M, Sichez JP, et al: Intraoperative mapping of the subcortical language pathways using direct stimulations. An anatomo-functional study. Brain 125:199-214, 2002

36. Duffau H, Denvil D, Capelle L: Long term reshaping of language, sensory, and motor maps after glioma resection: a new parameter to integrate in the surgical strategy. J Neurol Neurosurg Psychiatry 72:511-516, 2002

37. Duffau H, Denvil D, Lopes M, Gasparini F, Cohen L, Capelle $\mathrm{L}$, et al: Intraoperative mapping of the cortical areas involved in multiplication and subtraction: an electrostimulation study in a patient with a left parietal glioma. J Neurol Neurosurg Psychiatry 73:733-738, 2002

38. Duffau H, Gatignol P, Mandonnet E, Capelle L, Taillandier L: Intraoperative subcortical stimulation mapping of language pathways in a consecutive series of 115 patients with Grade II glioma in the left dominant hemisphere. J Neurosurg 109:461-471, 2008

39. Duffau H, Gatignol P, Mandonnet E, Peruzzi P, Tzourio-Mazoyer N, Capelle L: New insights into the anatomo-functional connectivity of the semantic system: a study using corticosubcortical electrostimulations. Brain 128:797-810, 2005 


\section{G. Bertani et al.}

40. Duffau H, Lopes M, Arthuis F, Bitar A, Sichez JP, Van Effenterre R, et al: Contribution of intraoperative electrical stimulations in surgery of low grade gliomas: A comparative study between two series without (1985-96) and with (1996-2003) functional mapping in the same institution. J Neurol Neurosurg Psychiatry 76:845-851, 2005

41. Ebel H, Ebel M, Schillinger G, Klimek M, Sobesky J, Klug $\mathrm{N}$ : Surgery of intrinsic cerebral neoplasms in eloquent areas under local anesthesia. Minim Invasive Neurosurg 43:192196,2000

42. Ebeling U, Schmid UD, Ying H, Reulen HJ: Safe surgery of lesions near the motor cortex using intraoperative mapping techniques: a report on 50 patients. Acta Neurochir (Wien) 119:23-28, 1992

43. Everhard S, Kaloshi G, Crinière E, Benouaich-Amiel A, Lejeune J, Marie Y, et al: MGMT methylation: a marker of response to temozolomide in low-grade gliomas. Ann Neurol 60:740-743, 2006

44. Fukaya C, Katayama Y, Yoshino A, Kobayashi K, Kasai M, Yamamoto T: Intraoperative wake-up procedure with propofol and laryngeal mask for optimal excision of brain tumor in eloquent areas. J Clin Neurosci 8:253-255, 2001

45. Galanaud D, Chinot O, Nicoli F, Confort-Gouny S, Le Fur Y, Barrie-Attarian M, et al: Use of proton magnetic resonance spectroscopy of the brain to differentiate gliomatosis cerebri from low-grade glioma. J Neurosurg 98:269-276, 2003

46. Gasparini FM, Cohen L, Lopes M, Denvil D, Capelle L, Duffau $\mathrm{H}$, et al: A clinical study of the number processing system: decimal size effects on reading numbers in patients with left parieto-occipital gliomas. Rev Neurol (Paris) 161:427-435, 2005

47. Giussani C, Roux FE, Lubrano V, Gaini SM, Bello L: Review of language organisation in bilingual patients: what can we learn from direct brain mapping? Acta Neurochir (Wien) 149:1109-1116, 2007

48. Goldstein B, Armstrong CL, Modestino E, Ledakis G, John C, Hunter JV: The impact of left and right intracranial tumors on picture and word recognition memory. Brain Cogn 54:1-6, 2004

49. Goldstein B, Obrzut JE, John C, Hunter JV, Armstrong CL: The impact of low-grade brain tumors on verbal fluency performance. J Clin Exp Neuropsychol 26:750-758, 2004

50. Goldstein B, Obrzut JE, John C, Ledakis G, Armstrong CL: The impact of frontal and non-frontal brain tumor lesions on Wisconsin Card Sorting Test performance. Brain Cogn 54:110-116, 2004

51. Gossl C, Fahrmeir L, Putz B, Auer LM, Auer DP: Fiber tracking from DTI using linear state space models: detectability of the pyramidal tract. Neuroimage 16:378-388, 2002

52. Guillevin R, Menuel C, Duffau H, Kujas M, Capelle L, Aubert A, et al: Proton magnetic resonance spectroscopy predicts proliferative activity in diffuse low-grade gliomas. J Neurooncol 87:181-187, 2008

53. Haglund MM, Berger M: Functional mapping of motor, sensory and language pathways during low-grade glioma removal. Tech Neurosurg 2:141-149, 1996

54. Haglund MM, Ojemann GA, Blasdel GG: Optical imaging of bipolar cortical stimulation. J Neurosurg 78:785-793, 1993

55. Heimans JJ, Taphoorn MJ: Impact of brain tumour treatment on quality of life. J Neurol 249:955-960, 2002

56. Hildebrand J, Lecaille C, Perennes J, Delattre JY: Epileptic seizures during follow-up of patients treated for primary brain tumors. Neurology 65:212-215, 2005

57. Hoang-Xuan K, Capelle L, Kujas M, Taillibert S, Duffau H, Lejeune J, et al: Temozolomide as initial treatment for adults with low-grade oligodendrogliomas or oligoastrocytomas and correlation with chromosome 1p deletions. J Clin Oncol 22:3133-3138, 2004

58. Holodny AI, Schulder M, Liu WC, Wolko J, Maldjian JA,
Kalnin AJ: The effect of brain tumors on BOLD functional MR Imaging activation in the adjacent motor cortex: implications for image-guided neurosurgery. AJNR Am J Neuroradiol 21:1415-1422, 2000

59. Jbabdi S, Mandonnet E, Duffau H, Capelle L, Swanson KR, Pelegrini-Issac M, et al: Diffusion tensor imaging allows anisotropic growth simulations of low grade gliomas. Magn Reson Med 54:616-624, 2005

60. Kaloshi G, Benouaich-Amiel A, Diakite F, Taillibert S, Lejeune J, Laigle-Donadey F, et al: Temozolomide for low-grade gliomas: predictive impact of $1 \mathrm{p} / 19 \mathrm{q}$ loss on response and outcome. Neurology 68:1831-1836, 2007

61. Kamada K, Todo T, Masutani Y, Aoki S, Ino K, Takano T, et al: Combined use of tractography-integrated functional neuronavigation and direct fiber stimulation. J Neurosurg 102:664-672, 2005

62. Keles GE: Intracranial neuronavigation with intraoperative magnetic resonance imaging. Curr Opin Neurol 17:497-500, 2004

63. Keles GE, Chang EF, Lamborn KR, Tihan T, Chang CJ, Chang $\mathrm{SM}$, et al: Volumetric extent of resection and residual contrast enhancement on initial surgery as predictors of outcome in adult patients with hemispheric anaplastic astrocytoma. J Neurosurg 105:34-40, 2006

64. Keles GE, Lamborn KR, Berger MS: Coregistration accuracy and detection of brain shift using intraoperative sononavigation during resection of hemispheric tumors. Neurosurgery 53:556-564, 2003

65. Keles GE, Lamborn KR, Berger MS: Low grade hemispheric gliomas in adults: a critical review of extent of resection as a factor influencing outcome. J Neurosurg 95:735-745, 2001

66. Keles GE, Lundin DA, Lamborn KR, Chang EF, Ojemann G, Berger MS: Intraoperative subcortical stimulation mapping for hemispherical perirolandic gliomas located within or adjacent to the descending motor pathways: evaluation of morbidity and assessment of functional outcome in 294 patients. J Neurosurg 100:369-375, 2004

67. Kleihues P, Cavenee W (eds): Pathology and Genetics of Tumours of the Nervous System. Lyon: IARC Press, 2000

68. Kleihues P, Louis DN, Scheithauer BW, Rorke LB, Reifenberger G, Burger PC, et al: The WHO classification of tumors of the nervous system. J Neuropathol Exp Neurol 61:215229, 2002

69. Klein M, Engelberts NH, van der Ploeg HM, Kasteleijn-Nolst Trenité DG, Aaronson NK, Taphoorn MJ, et al: Epilepsy in low-grade gliomas: the impact on cognitive function and quality of life. Ann Neurol 54:514-520, 2003

70. Klein M, Heimans JJ: The measurement of cognitive functioning in low-grade glioma patients after radiotherapy. J Clin Oncol 22:966-967, 2004

71. Klein M, Heimans JJ, Aaronson NK, Postma TJ, Muller M, van der Ploeg HM, et al: [Impaired cognitive functioning in low-grade glioma patients: relationship to tumor localisation, radiotherapy and the use of anticonvulsants.] Ned Tijdschr Geneeskd 148:2175-2180, 2004 (Dutch)

72. Kuznetsov YE, Caramanos Z, Antel SB, Preul MC, Leblanc $\mathrm{R}$, Villemure JG, et al: Proton magnetic resonance spectroscopic imaging can predict length of survival in patients with supratentorial gliomas. Neurosurgery 53:565-576, 2003

73. Lang FF, Gilbert MR: Diffusely infiltrative low grade gliomas in adults. J Clin Oncol 24:1236-1245, 2006

74. Laws ER, Shaffrey ME, Morris A, Anderson FA Jr: Surgical management of intracranial gliomas-does radical resection improve outcome? Acta Neurochir Suppl 85:47-53, 2003

75. Lucas TH, McKhann GM, Ojemann GA: Functional separation of languages in the bilingual brain: a comparison of electrical stimulation language mapping in 25 bilingual patients and 117 monolingual control patients. J Neurosurg 101:449457, 2004 
76. Mandonnet E, Delattre JY, Tanguy ML, Swanson KR, Carpentier AF, Duffau H, et al: Continuous growth of mean tumor diameter in a subset of grade II gliomas. Ann Neurol 53:524-528, 2003

77. Mandonnet E, Jbabdi S, Taillandier L, Galanaud D, Benali H, Capelle L, et al: Preoperative estimation of residual volume for WHO grade II glioma resected with intraoperative functional mapping. Neuro Oncol 9:63-69, 2007

78. Manninen PH, Tan TK: Postoperative nausea and vomiting after craniotomy for tumor surgery: a comparison between awake craniotomy and general anesthesia. J Clin Anesth 14:279-283, 2002

79. Mariani L, Siegenthaler P, Guzman R, Friedrich D, Fathi AR, Ozdoba $\mathrm{C}$, et al: The impact of tumour volume and surgery on the outcome of adults with supratentorial WHO grade II astrocytomas and oligoastrocytomas. Acta Neurochir (Wien) 146:441-448, 2004

80. Nakamura M, Konishi N, Tsunoda S, Nakase H, Tsuzuki T, Aoki $\mathrm{H}$, et al: Analysis of prognostic and survival factors related to treatment of low-grade astrocytomas in adults. Oncology 58:108-116, 2000

81. Neuloh G, Schramm J: Motor evoked potential monitoring for the surgery of brain tumours and vascular malformations. Adv Tech Stand Neurosurg 29:171-228, 2004

82. Nikas DC, Bello L, Zamani AA, Black PM: Neurosurgical considerations in supratentorial low-grade gliomas: experience with 175 patients. Neurosurg Focus 4(4):e4, 1998

83. Nimsky C, Ganslandt O, Hastreiter P, Wang R, Benner T, Sorensen AG, et al: Intraoperative diffusion-tensor MR imaging: Shifting of white matter tracts during neurosurgical procedures-initial experience. Radiology 234:218-225, 2005

84. Nimsky C, Ganslandt O, Hastreiter P, Wang R, Benner T, Sorensen AG, et al: Preoperative and intraoperative diffusion tensor imaging-based fiber tracking in glioma surgery. Neurosurgery 56:130-138, 2005

85. Ojemann G, Ojemann G, Lettich E, Berger M: Cortical language localization in left, dominant hemisphere. An electrical stimulation mapping investigation in 117 patients. J Neurosurg 71:316-326, 1989

86. Pallud J, Mandonnet E, Duffau H, Kujas M, Guillevin R, Galanaud D, et al: Prognostic value of initial magnetic resonance imaging growth rates for World Health Organization grade II gliomas. Ann Neurol 60:380-383, 2006

87. Pignatti F, van den Bent M, Curran D, Debruyne C, Sylvester $\mathrm{R}$, Therasse $\mathrm{P}$, et al: Prognostic factors for survival in adult patients with cerebral low-grade glioma. J Clin Oncol 20:2076-2084, 2002

88. Quinn JA, Reardon DA, Friedman AH, Rich JN, Sampson JH, Provenzale JM, et al: Phase II trial of temozolomide in patients with progressive low-grade glioma. J Clin Oncol 21:646-651, 2003

89. Rasmussen IA Jr, Lindseth F, Rygh OM, Berntsen EM, Selbekk T, Xu J, et al: Functional neuronavigation combined with intra-operative 3D ultrasound: initial experiences during surgical resections close to eloquent brain areas and future directions in automatic brain shift compensation of preoperative data. Acta Neurochir (Wien) 149:365-378, 2007

90. Reijneveld JC, Sitskoorn MM, Klein M, Nuyen J, Taphoorn MJ: Cognitive status and quality of life in patients with suspected versus proven low-grade gliomas. Neurology 56:618623,2001

91. Reinges MH, Nguyen HH, Krings T, Hutter BO, Rohde V, Gilsbach JM: Course of brain shift during microsurgical resection of supratentorial cerebral lesions: limits of conventional neuronavigation. Acta Neurochir (Wien) 146:369-377, 2004

92. Reithmeier T, Krammer M, Gumprecht H, Gerstner W, Lumenta CB: Neuronavigation combined with electrophysiological monitoring for surgery of lesions in eloquent brain areas in 42 cases: a retrospective comparison of the neurological outcome and the quality of resection with a control group with similar lesions. Minim Invasive Neurosurg 46:65-71, 2003

93. Romstock J, Fahlbusch R, Ganslandt O, Nimsky C, Strauss C: Localisation of the sensorimotor cortex during surgery for brain tumours: feasibility and waveform patterns of somatosensory evoked potentials. J Neurol Neurosurg Psychiatry 72:221229, 2002

94. Rostomily RC, Keles GE, Berger MS: Radical surgery in the management of low-grade and high-grade gliomas. Baillieres Clin Neurol 5:345-369, 1996

95. Roux FE, Boetto S, Sacko O, Chollet F, Tremoulet M: Writing, calculating, and finger recognition in the region of the angular gyrus: a cortical stimulation study of Gerstmann syndrome. J Neurosurg 99:716-727, 2003

96. Roux FE, Boulanouar K, Lotterie JA, Mejdoubi M, LeSage JP, Berry I: Language functional magnetic resonance imaging in preoperative assessment of language areas: correlation with direct cortical stimulation. Neurosurgery 52:1335-1345, 2003

97. Roux FE, Tremoulet M: Organization of language areas in bilingual patients: a cortical stimulation study. J Neurosurg 97:857-864, 2002

98. Roux FE, Lubrano V, Lauwers-Cances V, Tremoulet M, Mascott $\mathrm{CR}$, Demonet JF: Intra-operative mapping of cortical areas involved in reading in mono- and bilingual patients. Brain 127:1796-1810, 2004

99. Rozet I: Anesthesia for functional neurosurgery: the role of dexmedetomidine. Curr Opin Anaesthesiol 21:537-543, 2008

100. Rutten GJ, Ramsey N, Noordmans HJ, Willems P, van Rijen P, van der Berkelbach Sprenkel JW, et al: Toward functional neuronavigation: implementation of functional magnetic resonance imaging data in a surgical guidance system for intraoperative identification of motor and language cortices. Technical note and illustrative case. Neurosurg Focus 15(1):E6, 2003

101. Rutten GJ, Ramsey NF, van Rijen PC, Noordmans HJ, van Veelen CW: Development of a functional magnetic resonance imaging protocol for intraoperative localization of critical temporo-parietal language areas. Ann Neurol 51:350-360, 2002

102. Sanai N, Berger MS: Glioma extent of resection and its impact on patient outcome. Neurosurgery 62:753-756, 2008

103. Sarang A, Dinsmore J: Anesthesia for awake craniotomy-evolution of a technique that facilitates awake neurological testing. Br J Anaesth 90:161-165, 2003

104. Schmidt MH, Berger MS, Lamborn KR, Aldape K, McDermott MW, Prados MD, et al: Repeated operations for infiltrative low-grade gliomas without intervening therapy. J Neurosurg 98:1165-1169, 2003

105. Smith JS, Chang EF, Lamborn KR, Chang SM, Prados MD, Cha $\mathrm{S}$, et al: Role of extent of resection in the long-term outcome of low-grade hemispheric gliomas. J Clin Oncol 26:1338-1345, 2008

106. Smith JS, Perry A, Borell TJ, Lee HK, O'Fallon J, Hosek SM, et al: Alterations of chromosome arms $1 p$ and $19 q$ as predictors of survival in oligodendrogliomas, astrocytomas, and mixed oligoastrocytomas. J Clin Oncol 18:636-645, 2000

107. Souter MJ, Rozet I, Ojemann JG, Souter KJ, Holmes MD, Lee L, et al: Dexmedetomidine sedation during awake craniotomy for seizure resection: effects on electrocorticography. J Neurosurg Anesthesiol 19:38-44, 2007

108. Stupp R, Janzer RC, Hegi ME, Villemure JG, Mirimanoff RO: Prognostic factors for low-grade gliomas. Semin Oncol 30:23-28, 2003

109. Szelényi A, Langer D, Beck J, Raabe A, Flamm ES, Seifert $\mathrm{V}$, et al: Transcranial and direct cortical stimulation for motor evoked potential monitoring in intracerebral aneurysm surgery. Neurophysiol Clin 37:391-398, 2007

110. Talos IF, Zou KH, Ohno-Machado L, Bhagwat JG, Kikinis R, Black PM, et al: Supratentorial low-grade glioma resectability: statistical predictive analysis based on anatomic MR features and tumor characteristics. Radiology 239:506-513, 2006 


\section{G. Bertani et al.}

111. Taphoorn MJ: Neurocognitive sequelae in the treatment of lowgrade gliomas. Semin Oncol 30:45-48, 2003

112. Van den Bent MJ, Looijenga LH, Langenberg K, Dinjens W, Graveland W, Uytdewilligen L, et al: Chromosomal anomalies in oligodendroglial tumors are correlated with clinical features. Cancer 97: 1276-1284, 2003

113. van Veelen ML, Avezaat CJ, Kros JM, van Putten W, Vecht C: Supratentorial low grade astrocytoma: Prognostic factors, dedifferentiation, and the issue of early versus late surgery. J Neurol Neurosurg Psychiatry 64:581-587, 1998

114. Vlieger EJ, Majoie CB, Leenstra S, den Heeten GJ: Functional magnetic resonance imaging for neurosurgical planning in neurooncology. Eur Radiol 14:1143-1153, 2004

115. Whittle IR, Midgley S, Georges H, Pringle AM, Taylor R: Patient perceptions of "awake" brain tumour surgery. Acta Neurochir (Wien) 147:275-277, 2005
116. Yingling CD, Ojemann S, Dodson B, Harrington MJ, Berger MS: Identification of motor pathways during tumor surgery facilitated by multichannel electromyographic recording. J Neurosurg 91:922-927, 1999

Manuscript submitted June 14, 2009.

Accepted August 5, 2009.

Address correspondence to: Lorenzo Bello, M.D., Neurochirurgia, Dipt. di Scienze Neurologiche, Università degli Studi di Milano, Fondazione IRCCS Ospedale Maggiore Policlinico, Mangiagalli e Regina Elena Via Francesco Sforza 35, 20122, Milan, Italy. email: lorenzo.bello@unimi.it. 